\title{
The cardiomyopathy associated with methylsalicylate
}

\author{
Hillary P. OJiambo \\ M.D., F.R.C.P. \\ Department of Medicine, University of Nairobi, Kenya
}

\begin{abstract}
Summary
Dogs challenged with toxic doses of methylsalicylate developed acute myocardiopathy. Metabolic manifestations of uncoupling of oxidative phosphorylation by methylsalicylate (MS) were observed. Oxygen consumption increased two-fold. The haemodynamic abnormalities produced by intervention were minimal. The measured metabolic parameters indicated profound derangement. A net efflux of orthophosphate, lactate and potassium was observed from the myocardium. Marked hyperkalaemia occurred terminally. Morphological changes were observed on electron microscopy.
\end{abstract}

\section{Introduction}

Methylsalicylate (MS) is known to uncouple oxidative phosphorylation (Brody, 1956). The possible effects of this agent on myocardial mechanics and metabolism have not been extensively studied. The death of two patients following the accidental ingestion of MS prompted this study. Their electrocardiograms showed evidence of hyperkalaemia (Fig. 1) and this could not be reversed by the usual methods. Autopsy revealed fragmentation and, in one case, frank myocytolysis was also observed. This study was designed to answer the following questions:

(1) Is myocardial failure due to MS toxicity due to pump failure, or is it purely a metabolic phenomenon?

(2) What are the mechanisms leading to a net efflux of potassium, and a high extracellular $\mathrm{K}^{+}$?

(3) What is the role of lactic acidosis in MSinduced myopathy?

(4) What significance should be attached to the observed changes in myocardial extraction of oxygen, elevated levels of creatine-phosphokinase (CPK) in the coronary effluent and a net loss of inorganic phosphate?

\section{Material and methods}

Healthy mongrel dogs (mean weight $12.5 \mathrm{~kg}$ ) with a known metabolic history and in a fasting state were lightly anaesthetized with pentobarbital sodium. The left femoral artery was catheterized retrograde using a No. 6 USCI end-hold catheter. Systemic blood pressure was monitored with the Bell and Howell transducers connected to a Sanborn multichannel recorder. The coronary sinus was intubated using a No. 5 RA catheter under fluoroscopic guide. The position of the catheter was ascertained by dye injection, coupled with the findings of a persistently low $\mathbf{P O}_{2}$. The left ventricle was catheterized from the internal carotid with a No. 7 side hole cordis catheter and connected to the Sanborn recorder. Ventriculography was done to check the exact position of the catheter. Advantage was taken of this manoeuvre to make pertinent observations of the myocardial contractility and clearance of dye from the left ventricle. A standard lead II ECG was taken using skin electrodes. Catheters were also placed in both femoral veins and the contralateral femoral artery for sampling of metabolites and pressure measurements. Following a control period, MS was administered intragastrically in the dosage of 700 $\mathrm{mg} / \mathrm{kg}$. Experimental procedures were carried out over a 4-5 hr period, at which time the preparations died or were sacrificed.

Blood gases, $\mathrm{pH}, \mathrm{PCO}_{2}, \mathrm{PO}_{2}$ were measured using an IL electrode. Oxygen saturation by oximetry. Lactate was measured by the enzymatic method of Horn and Bruns (1956). Potassium was determined by flame photometry using internal standards. Creatine phosphokinase was estimated by the method of Kuby, Norda and Lardy (1954). Plasma inorganic phosphate was measured by a modification of the method of Kuttner and Cohen (1927). The cardiac output was determined by the Fick method. The total plasma salicylate level was measured in arterial blood using the Stevenson (1960) method.

\section{Histochemical methods}

Portions of myocardium were quenched in isopentane, precooled in liquid nitrogen. Sections $10 \mu$ in thickness were cut in a cryostat and mounted on coverslips. For the visualization of glycogen, sections were fixed in cold Gendre's solution. Sections for enzymatic incubations were air-dried and those used for the demonstration of potassium were freeze-dried for $1 \mathrm{hr}$ before staining. Glycogen was detected by PAS stain. Control sections were pretreated with 

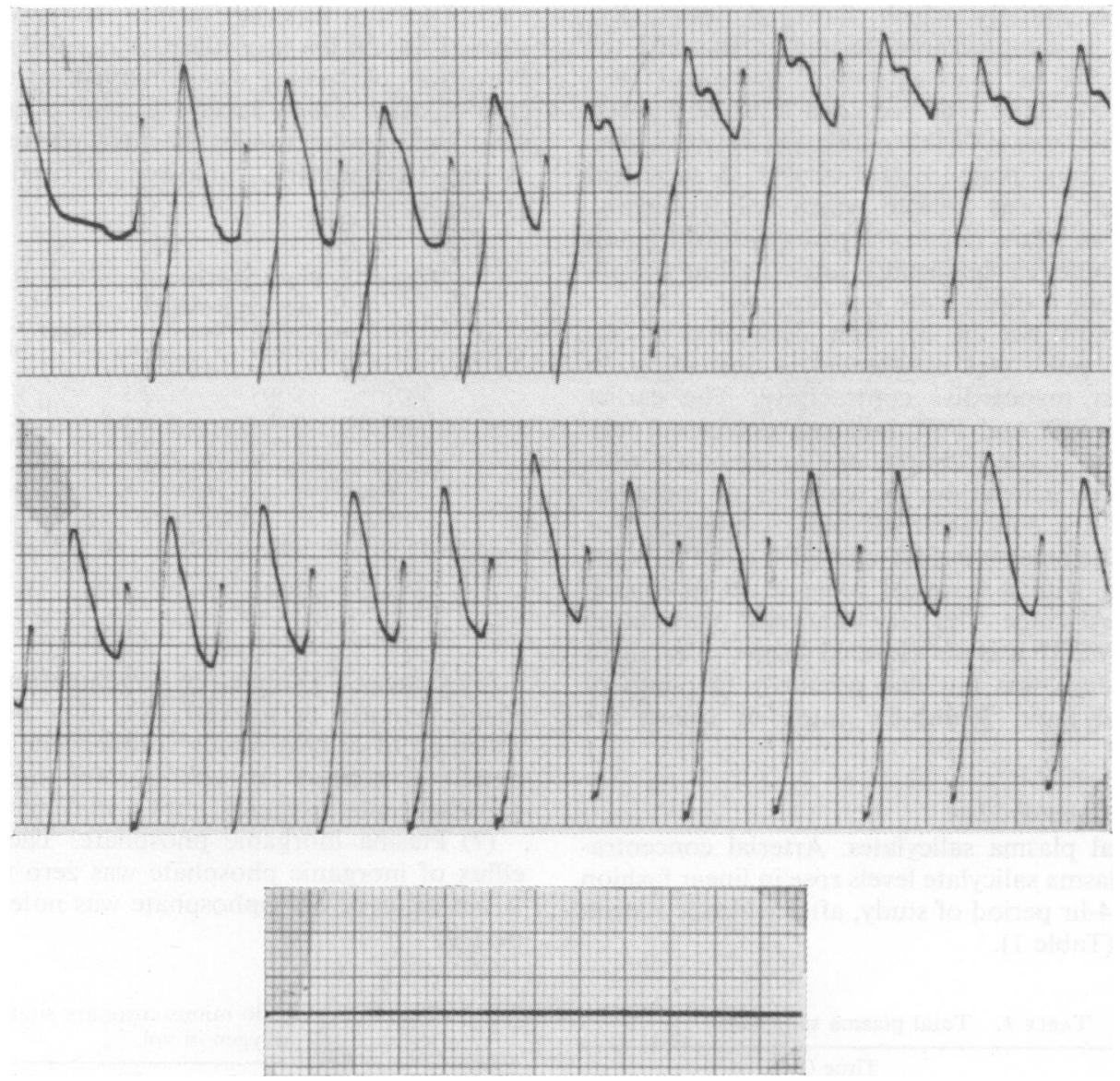

FIG. 1. Electrocardiograms of a patient who ingested methylsalicylate. Note the widening of $Q R S$ complexes and peaked $T$ waves.

diastase. Succinic dehydrogenase, lactate dehydrogenase, and $\beta$-hydroxybutyrate dehydrogenase were determined using a standard method (Pearse, 1961). Freeze-dried floating sections were stained for the detection of potassium by the method of Poppen et al. (1953) and Amon (1968).

\section{Electron microscopy}

The hearts were perfused immediately after the death or sacrifice. Karnovsky (1965) fluid modified by the addition of $5 \%$ sucrose was used as the perfusate. The preservative was perfused through a cannula inserted into the anterior descending or circumflex branch of the left coronary artery. Tissue blocks were excised from the perfused areas of the myocardium. They were fixed in glutaraldehydeparaformaldehyde mixture, and post-fixed in $1 \%$ osmium tetroxide, and, thereafter, dehydrated in graded ethanols and embedded in Epon 912. Silver sections from selected areas of the myocardium were cut and stained by uranyl acetate and lead nitrate according to the method of Venable and Coggeshall (1965).

In utilizing the technique of coronary sinus intubation in the study of myocardial metabolism, it was assumed that all the venous drainage from the left ventricle was by the way of the coronary sinus. Evidence indicates that this is true in respect of man and the dog although the coronary effluent has been found not homogeneous (Neil, 1968).

\section{Results}

\section{Myocardial mechanics and haemodynamics}

The mechanical parameters measured were: (a) the systolic ejection time (SET); (b) time to peak tension (PTT); (c) the left ventricular end-diastolic pressure (LVEDP).

The left ventricular pulse contour showed an 
abrupt rise initially which flattened terminally. There was a demonstrable decline in the SET of $25 \%$ at $3-3.5 \mathrm{hr}$, but a similar increment was observed terminally. The recorded LVEDP showed no rise, the mean LVEDP oscillating between zero and a maximum pressure of $5 \mathrm{mmHg}$. A sustained cardiac output was noticed which fell terminally. There was an initial rise in the mean systemic blood pressure which fell significantly after the 4-hr period, at which time a shock state was observed.

The abrupt rise in the first derivative of the ventricular pulse was interpreted as indicating an increase in myocardial contractility. The earlier decrease in SET and PTT indicates decreased peripheral resistance or after load and/or increased myocardial force generation. A tendency to increased activity of the contractile element is inferred. The observed increments in SET and PTT terminally are in keeping with a terminal increase in peripheral vascular resistance. The very important observation that the LVEDP was not elevated suggests that myocardial failure was not due primarily to pump or mechanical fault. Metabolic cause or causes are postulated.

\section{Myocardial metabolism}

(1) Total plasma salicylates. Arterial concentrations of plasma salicylate levels rose in linear fashion up to the 4-hr period of study, after which a plateau occurred (Table 1).

TABLE 1. Total plasma salicylate

\begin{tabular}{lrrrrr}
\hline \multicolumn{5}{c}{ Time (hr) } \\
\hline Mean & 12.2 & 19.6 & 29.9 & $41 \cdot 3$ & 37.6 \\
s.e. mean & \pm 1.3 & \pm 2.7 & \pm 3.4 & \pm 6.6 & \pm 6.9 \\
\hline
\end{tabular}

Mean \pm s.e. mean plasma salicylate levels measured in arterial blood throughout the study.

(2) Creatine phosphokinase. A rise in CPK was observed in the coronary effluent. A mean arterial level of 12.5 sigma units was recorded after $2 \mathrm{hr}$ (normal 4 units). The rise in the level of CPK is indicative of myocardial cell damage. The specificity of CPK as an index of muscle damage has been stressed (Wexler, 1970; Lanari, Perez and Semenink, 1970).

(3) Acid-base changes. A period of respiratory alkalosis was initially observed followed after the third $\mathrm{hr}$ by a metabolic acidosis.

(4) Potassium. A gradual rise in the arterial concentration of potassium was noted. The arterial minus coronary sinus difference for potassium showed a net uptake of $\mathrm{K}^{+}$up to the third hour of study. Thereafter, a net efflux of $\mathrm{K}^{+}$was observed (Fig. 1).
(5) Lactate. Arterial lactate concentration increased during the procedure. A normal myocardial extraction of lactate was observed up to the $2-\mathrm{hr}$ period, after which lactate output occurred. The mean lactate extraction by the myocardium expressed as $\mathrm{A}-\mathrm{Cs} / \mathrm{A} \times 100 \%$ followed a similar pattern (Table 2).

TABLE 2. Per cent extraction of metabolites by the myocardium

\begin{tabular}{lccc}
\hline & Control & $2-\mathrm{hr}$ & $4-\mathrm{hr}$ \\
\hline $\mathrm{PO}_{4}^{--}$ & +0.9 & $-2 \cdot 3$ & $-3 \cdot 1$ \\
Lactate & +8.9 & -3.1 & -55.8 \\
$\mathrm{~K}^{+}$ & +1.7 & +4.8 & -16.8 \\
\hline
\end{tabular}

Mean per cent extraction by the myocardium of phosphate, lactate, and potassium at control, $2 \mathrm{hr}$ and $4 \mathrm{hr}$ $\left(\frac{\mathrm{A}-\mathrm{Cs}}{\mathrm{A}} \times 100 \%\right)$

(6) Oxygen. Despite hyperventilation, only a slight increase in arterial $\mathrm{PO}_{2}$ was observed. A widening of arterio minus coronary sinus $\mathrm{PO}_{2}$ was noted. Expressed in vol. \%, the arterio minus coronary sinus oxygen is shown in Table 3.

(7) Plasma inorganic phosphate. The mean net efflux of inorganic phosphate was zero up to $2 \mathrm{hr}$. A net efflux of orthophosphate was noted after this period.

Table 3. Arterio minus coronary sinus oxygen in vol. $\%$

\begin{tabular}{lrcccc}
\hline & Baseline & $1 \mathrm{hr}$ & $2 \mathrm{hr}$ & $3 \mathrm{hr}$ & $4 \mathrm{hr}$ \\
\hline Mean & 4.7 & 5.5 & 7.0 & 7.2 & 7.5 \\
s.e. mean & \pm 0.9 & \pm 1.1 & \pm 0.2 & \pm 0.10 & \pm 0.09 \\
\hline \multicolumn{5}{r}{ Mean and s.e. mean of A-Cs oxygen in vol. $\%$}
\end{tabular}

\section{Morphology}

No signs of microvascular occlusion were demonstrated. The staining method for potassium (Amon, 1968) did not reveal obvious diffuse or focal changes. Swelling of cardiac muscle cells with the separation of myofibrils and bulging or sarcolemma was observed (Fig. 2). Clear spaces suggesting dilatation of sarcoplasmic reticulum were noted (Fig. 3). Abnormalities were noted in the mitochondria. These were focal areas of clearing of the matrix. In some mitochondria vacuolation and bulging of the outer limiting membrane occurred (Fig. 4). It is noted however that these changes were discrete and focal.

\section{Discussion}

The observed changes suggest the following hypothesis for the mode of action of MS. By uncoupling 


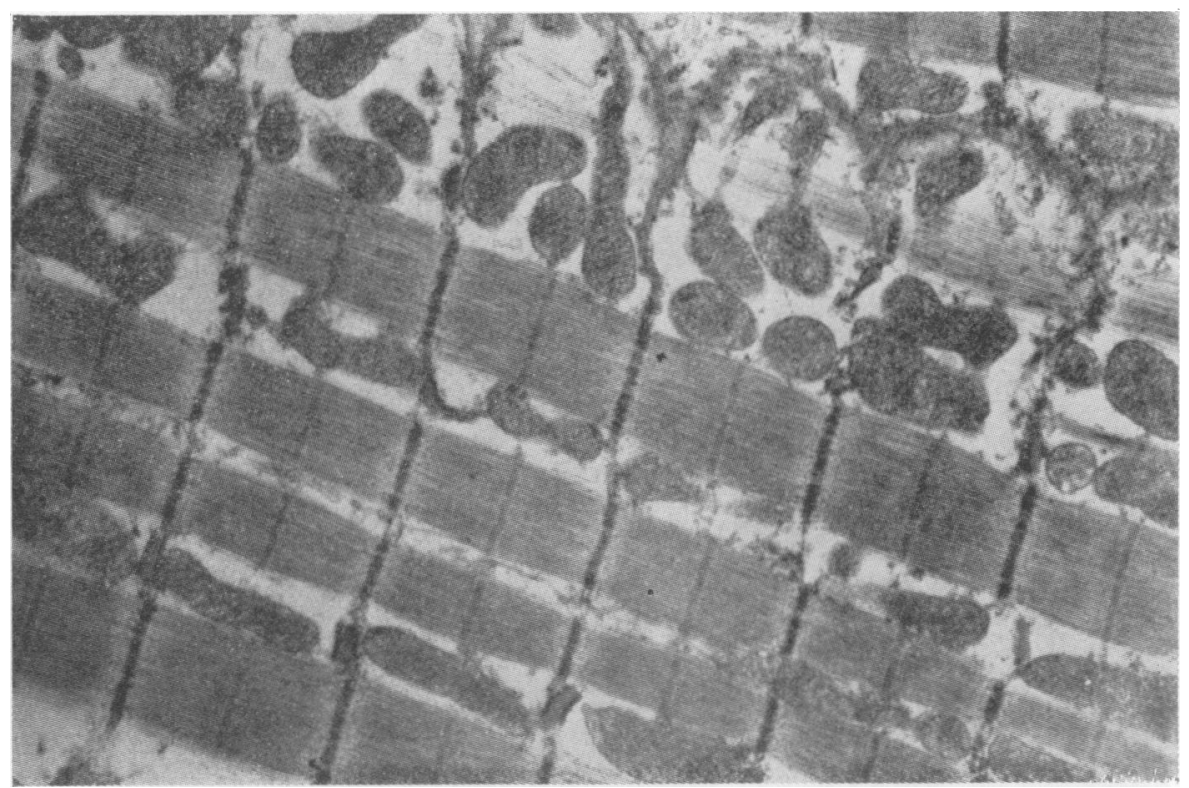

FIG. 2. Electronmicrograph showing cardiac muscle cell swelling with separation of myofibrils and bulging of the sarcolemma between the $T$ tubules $(\times 12,400)$.

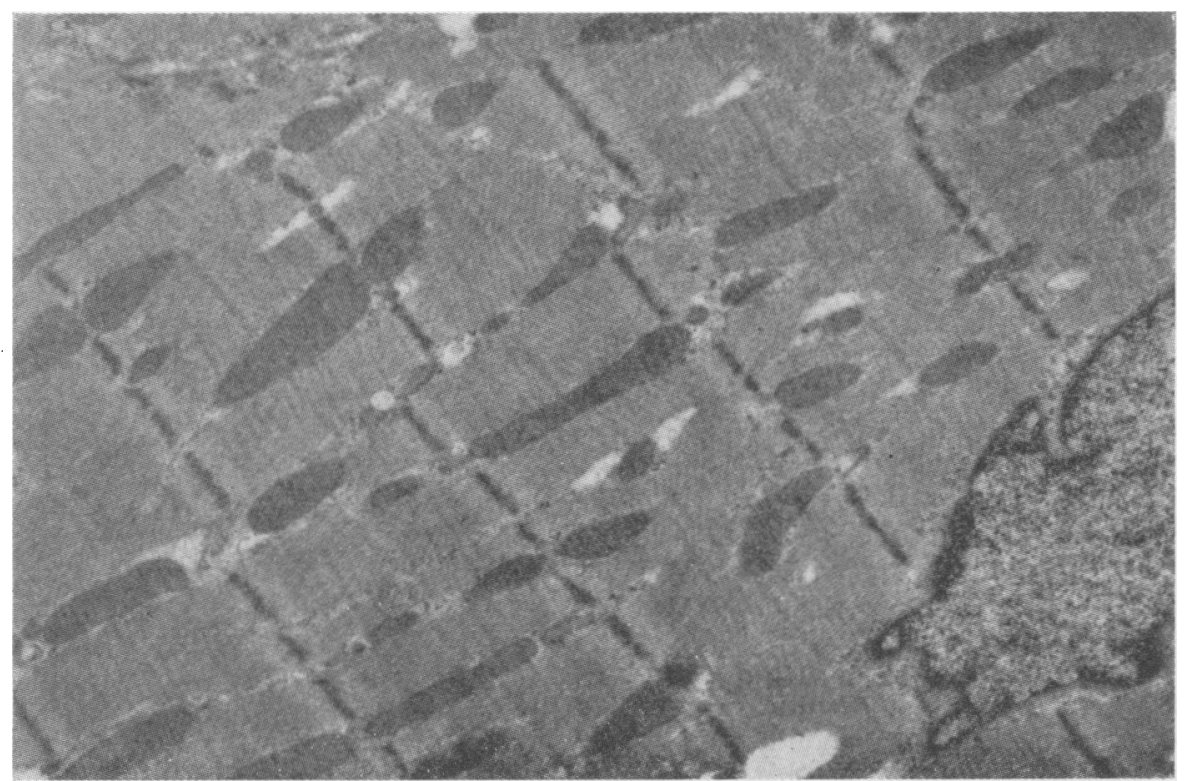

FIG. 3. Section through a part of cardiac muscle cell showing swelling. The clear spaces represent distended tubules of the sarcoplasmic reticulum $(\times 12,640)$. 


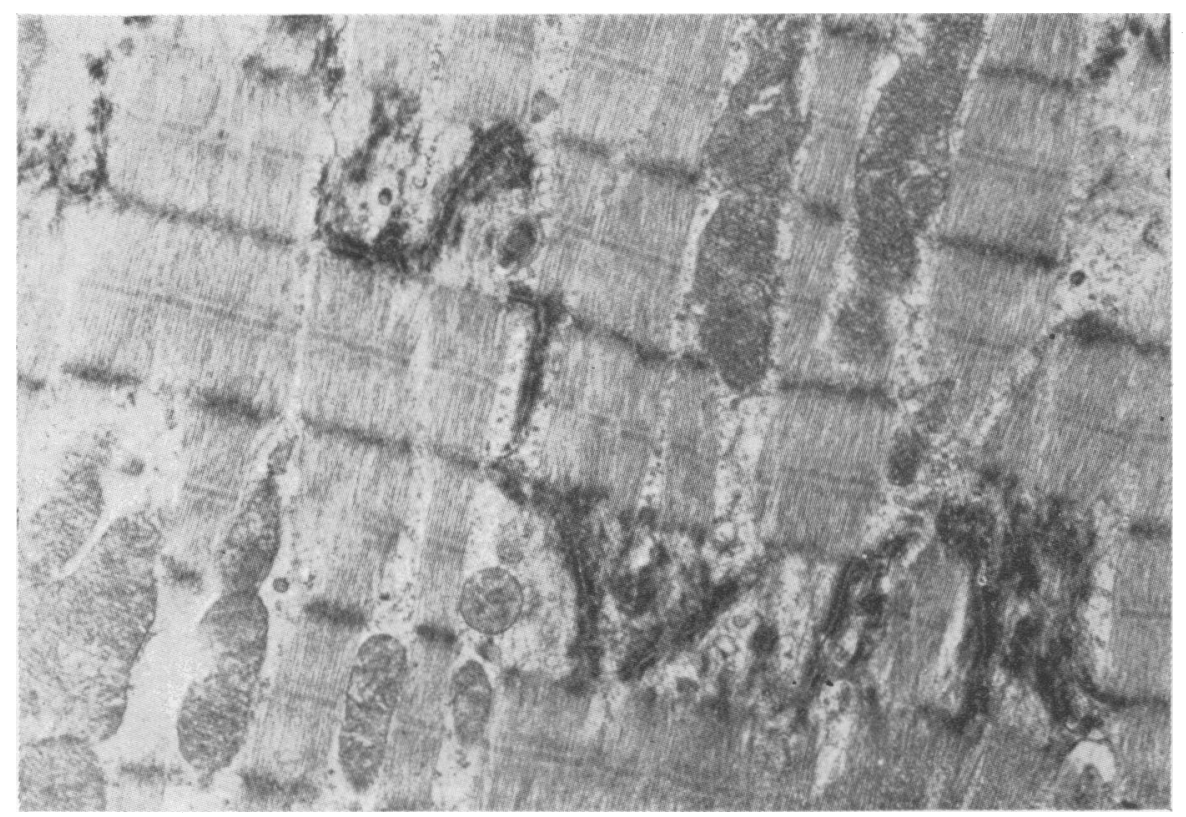

FIG. 4. Photomicrograph showing an area of an intercalated disc with swollen mitochondria and clearing of their matrix. A bleb is seen under the outer limiting membrane of one of the mitochondria in the lower left corner $(\times 11,680)$.

oxidative metabolism, MS interferes with the production of high energy phosphate. Inorganic phosphate and phosphate nucleotides accumulate. It has been shown (Newsholme and Randle, 1962) that these compounds stimulate glycolysis by activating phosphofructokinase. Lactate output from the myocardium occurred as a result of stimulation of glycolysis. The sodium pump requires ATP and the presence of $\mathrm{Na}^{+} / \mathrm{K}^{+}$activated, magnesium dependent ATPase to maintain normal potassium influx. Decreased availability of ATP, resulting from uncoupling, leads to a decrease in $\mathrm{K}^{+}$influx, and a dominant $\mathrm{K}^{+}$efflux from the myocardial cell, leading to an increase in extracellular concentrations of $\mathrm{K}^{+}$. Hyperkalaemia is known to reduce the resting membrane potential. The decrease in the transmembrane potential was responsible for the production of arrhythmias and terminally a cardiac standstill and death.

\section{Conclusion}

A model of an acute myocardiopathy has been described utilizing an uncoupling agent. Minimal haemodynamic changes occurred. Mechanical abnormalities were due to ionotropic action of salicylate in small concentrations. Increased concentration could be responsible for depressed myocardial contractility. Profound metabolic derangements were noted. These derangements are probably due to a lack of ATP production. Morphological changes could result from metabolic insufficiency, leading to inability of a cell to behave as an ideal osmometer. This could lead to imbibition of water and swelling of the cell and subcellular organelles. Information should be sought as to whether this form of toxic process occurring in man on a chronic basis could give rise to discrete myocardial scarring as is often seen in some forms of the so-called 'idiopathic' cardiomyopathies. These form the bulk of cardiological practice in mid-Africa. A plea is made for a metabolic approach in an endeavour to unravel the basic pathophysiology of these cardiomyopathies.

\section{Acknowledgments}

This work was supported by the Canadian Muscular Dystrophy Association and Canadian International Agency for Development and it is published with permission of the Editor of the African Journal of Medical Sciences. The experimental procedures were carried out at the McGill University Clinic of the Royal Victoria Hospital in the Department of Cardiology. I am indebted to Professor G. A. Klassen for valuable advice and to Mrs E. Summers for technical help.

\section{References}

Amon, H. (1968) Methodische Untersuchungen zum histochemischen Nachweis von Elektrolyten. II. Die lichtmikroskopische Darstellung von $\mathrm{K}^{+}$in der Niere. Histochemia, 14, 251. 
Brody, T.M. (1956) The action of sodium salicylate and related compounds on tissue metabolism in vitro. Journal of Pharmacology and Experimental Therapeutics, 117, 29.

HORN, H.D. \& BRUNS, F.H. (1956) Quantitative Bestimmung von $\mathbf{L}(+)$ Milschsäure mit Milchsäuredehydrogenäse. Biochimica et Biophysica acta, 21, 378.

KARNOVSKY, M.J. (1965) A formaldehyde-glutaraldehyde fixative of high osmolarity for the use in electronmicroscopy. Journal of Cell Biology, 27, 137A.

Kuby, S. A., Noda, L. \& Lardy, H.A. (1954) Adenosine triphosphate-creatine transphorylase. Journal of Biological Chemistry, 210, 65.

KutTner, T. \& COHEN, H.R. (1927) Micro colorimetric studies. Molybdic acid, stannous chloride reagent. The micro estimation of phosphate and calcium in pus, plasma and spinal fluid. Journal of Biological Chemistry, 75, 517.

Lanari, A., Perez, M.G. \& SemeninK, G.B. (1970) Increased levels of creatinine phosphokinase after transient limb ischaemia in patients with muscular dystrophy. Lancet, $\mathbf{i}$, 217.
NeIL, W.A. (1968) Myocardial hypoxia and metabolism in coronary heart disease. American Journal of Cardiology, 22, 507.

Newsholme, E.A. \& Randle, P.J. (1962) Regulation of glucose uptake by muscle. 6 Fructose, 1,6-diphosphatase of rat heart and rat diaphragm. Biochemistry, 83, 387.

PeARSE, A.G.E. (1960) Histochemistry Theoretical and Applied, 2nd edn, p. 910. Churchill, London.

Stevenson, G.W. (1960) Rapid ultraviolet spectrophotometric determination of salicylate in blood. Journal of Analytical Chemistry, 32, 1522.

Venable, J.H. \& Coggeshall, R. (1965) A simplified lead citrate stain for use in electron microscopy. Journal of Cell Biology 25, 407.

WEXLER, B.C. (1970) Serum creatine phosphokinase activity following isoproterenol-induced myocardia infarction in male and female rats with or without atherosclerosis. American Heart Journal, 79, 69.

\section{Discussion}

Chairman: Is there any discussion on this paper?

PROFESSOR HARRIS: It will not take much damage, if you call it damage, for any muscle to lose potassium; after all, if you exercise your leg, potassium pours out of the muscle.

Dr Ojiambo: Potassium leak is in fact one of the first signs of myocardial damage following coronary occlusion.

PROFESSOR HARRIS: It is a very, very early sign I think.

DR OLSEN: The only comment I would like to make is that it is always dangerous to judge from a few slides, but you mentioned the word myocytolysis, and on the evidence that you have shown, this was by no means marked; what I understand by myocytolysis is the disappearance of myofibrils or myocardial fibres. Now all you have shown us was that there was separation of some myofibrils, which were absolutely regularly arranged. If these changes can be produced by altering the power of resolution, or by changing fixation, I think that on the evidence you have shown here today, we cannot really talk about myocytolysis. Now with regard to the mitochondria, these little 'bulges' may sometimes be seen. I would consider crystolysis as more important.
Dr OJiambo: My answer to that is simply that these lesions were focal and discrete.

Dr OLSEN: Yes, but you did not show us sufficiently abnormal areas to permit the term myocytolysis to be used. I do not know what my colleagues of pathology would think. Your illustrations did not convince me of myocytolysis.

DR SCHWARTZ: I do not really see how anyone can make a judgment about mitochondria on the basis of pictures alone, unless he shows a million pictures, you can see any form of mitochondria; so I would take this as presumptive evidence only. You can still have a very significant recovery of oxidative phosphorylation and your pictures may give the impression that they are in perfectly normal mitochondria; I don't know what is normal and what is abnormal in your pictures.

DR OLSEN: I agree, but the only point I wish to make is that I know what I mean by abnormal changes, which when they are demonstrated (possibly selected from numerous preparations) would leave no doubt that they differ sufficiently from those changes which are accepted by most morphologists as normal. 